УДК 538.945

\title{
Fractal Boundaries of Vortex Pinning Clusters in Copper-oxide Superconductors in Magnetic Field
}

\author{
Mikhael A. Vasyutin* \\ Nikolay D. Kuzmichev \\ Dmitri A. Shilkin
}

Sub-Department of General Scientific Disciplines Mordovian Ogarev State University, Bolshevistskaya, 68 Saransk, 430005

Russia

Received 22.09.2016, received in revised form 16.01.2017, accepted 06.02.2017

The influence of fractal boundaries of a normal phase clusters on the flux creep in the copper-oxide hightemperature superconductors YBCO was investigated. A model of the magnetic-field dependence of the voltage due to flux creep for different transport currents was developed. To describe the experimental curves is proposed to use an exponential-hyperbolic function in which the argument is direct current. Empirical dependence of the fractal dimension of the cluster boundaries on the magnetic field strength was determined. The characteristic values of the magnetic field were found. The index of connectivity of the flux creep paths at the percolation threshold was calculated.

Keywords: superconductivity, normal phase clusters, fractal boundaries, flux creep. DOI: 10.17516/1997-1397-2017-10-2-261-265.

The boundaries of different areas in samples of weakly coupled superconductors play an important role, resulting in both a decrease and an increase of the critical current. The network of weak links has a fractal structure as in the whole volume of the sample and on the boundaries of various defects or inclusions. Fractal properties have been found in many copper-oxide hightemperature superconductors (HTSC) experimentally based on resistive measurements [1,2] and the direct supervision of using a magneto-optical technique [3-5]. Modeling of properties copperoxide HTSC is also possible with the help consideration of their fractal features [6-8].

Magnetic field parameters peculiarities the copper-oxide HTSC depend on at least of three factors: 1) distribution of inhomogeneities on the areas, 2) changes in the boundaries of these inhomogeneities, 3) changes in the network of weak links throughout the sample. All of these factors significantly associated with the fractal properties of the boundaries of the normal phase clusters. Such clusters are formed as by irradiating the samples with heavy ions, and during film growth or sintering of polycrystalline bulk samples [8-11].

The peculiarities of the normal phase clusters were considered in detail in papers [12-15]. In the article [8] it was found that the DC magnetic field strongly influences on the fractal dimension of the boundaries of the normal phase clusters. This is due to the formation of weak links and structural defects generated easily in HTSC because of the short coherence length in these materials. With increasing field strength from $\approx 50$ Oe to $\approx 200$ Oe fractal dimension increased from 1.2 to 1.9. Determination of the fractal dimension was done by fitting of the

*vasyutinm@mail.ru

(C) Siberian Federal University. All rights reserved 
experimental dependences of the first harmonic voltage from DC (proportional to the differential resistance at low currents) with help exponential-hyperbolic function. Parameter of this function was the fractal dimension.

In this paper we investigate the magnetic field dependence of the first harmonic of the voltage at different currents. Here direct current uses as a parameter, and the magnetic field dependence is mediated by fractal dimension of the boundaries of the normal phase clusters.

Samples of the copper-oxide HTSC $\mathrm{YBa}_{2} \mathrm{Cu}_{3} \mathrm{O}_{7-x}$ (YBCO) were prepared by the standard method of solid-phase synthesis of initial oxides. The density of polycrystalline samples was $4.18 \mathrm{~g} / \mathrm{cm}^{3}$. The temperature of the superconducting transition was $T_{c} \approx 92.0 \mathrm{~K}$. The transition width was $\Delta \mathrm{T} \approx 1.5 \mathrm{~K}$. Investigations were carried out at a temperature $\mathrm{T} \approx 77 \mathrm{~K}$ with help of four-contacts method. Contact resistance was less than $3 \mathrm{~m} \Omega$. Through the current contacts are skipped DC and AC currents. From potential contacts was measured voltage. The first voltage harmonic was allocated by selective amplifier. More measurement technique in paper $[16]$ is described.

Fig. 1 shows the dependence of the fractal dimension of the boundaries of the normal phase clusters D on strength of DC magnetic field $\mathrm{H}$. The dependence $\mathrm{D}(\mathrm{H})$, obtained in [8] (black circles), was approximated by the function (solid line):

$$
D=2 /\left[\exp \left(-\left(H-H_{1}\right) / H_{0}\right)+1\right]
$$

where $\mathrm{H}_{0}=40$ Oe and $\mathrm{H}_{1}=36$ Oe are the field strengths, is the characteristic of the given sample.

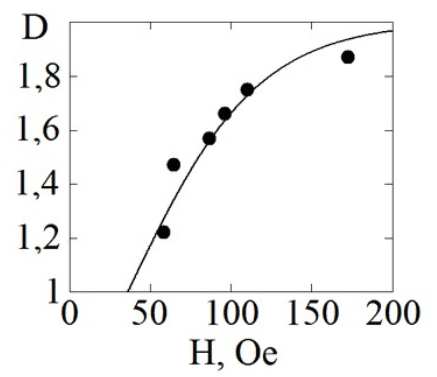

Fig. 1. The dependence of the fractal dimension of the normal phase clusters on the magnetic field strength. Black circles are the data obtained in paper [8], solid line is empirical dependence (equation (1))

Fig. 2 shows the experimental dependence of the amplitudes of the first voltage harmonic $\mathrm{V}_{1}$ for YBCO sample on the magnetic field strength (black circles) for different values of DC. The approximation $\mathrm{V}_{1}(\mathrm{H})$ is made by using an exponential-hyperbolic function found for $\mathrm{V}_{1}(\mathrm{i})$ of the same sample in paper [8]:

$$
V_{1}=R_{d} I_{a}=I_{a}\left[R_{d 1} \exp \left(-C_{1} C_{0} i^{-\mu}\right)+R_{d 2} \exp \left(-C_{2} C_{0} i^{-\mu}\right)\right]
$$

where $\mathrm{i}=\mathrm{I}_{d} / \mathrm{I}_{c}$ is the relative current, $\mathrm{I}_{d}$ is the $\mathrm{d}_{c}$ current, $\mathrm{I}_{c}=316 \mathrm{~mA}$ is the critical current at $\mathrm{H}=0, \mathrm{R}_{d}=\left(\mathrm{dV} / \mathrm{dI}_{d}\right)$ is the differential resistance, $\mathrm{I}_{a}=27 \mathrm{~mA}$ is the amplitude of the alternating current, $\mathrm{C}_{0}=(1+1 / \mu)^{\mu+1}, \mu=2 / \mathrm{D}(\mathrm{H})$ is the glassy state index [15], $\mathrm{C}_{1}=0.02, \mathrm{C}_{2}=0.14$, $\mathrm{R}_{d 1}=0.012 \mathrm{~m} \Omega, \mathrm{R}_{d 2}=0.78 \mathrm{~m} \Omega$. 


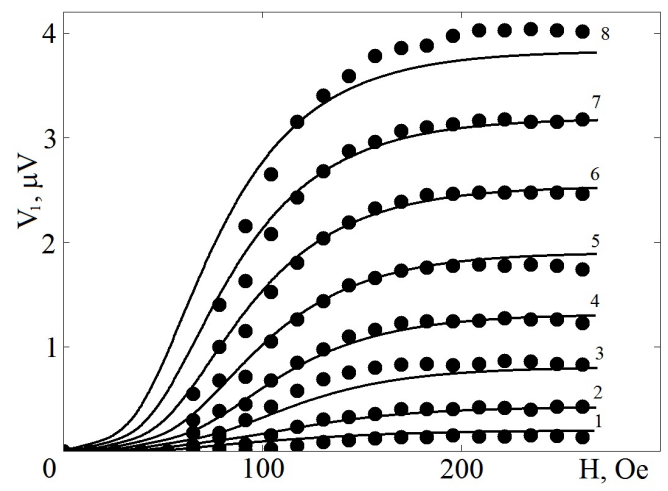

Fig. 2. Dependence of the amplitudes of the first voltage harmonic on magnetic field for different values of DC. Black circles are the experimental dependences, solid lines are the theoretical curves calculated by the formula (2). The numerals represent different values DC: $\mathrm{I}_{d}=30 \mathrm{~mA}(1), \mathrm{I}_{d}=$ $40 \mathrm{~mA}(2), \mathrm{I}_{d}=50 \mathrm{~mA}(3), \mathrm{I}_{d}=60 \mathrm{~mA}(4), \mathrm{I}_{d}=70 \mathrm{~mA}(5), \mathrm{I}_{d}=80 \mathrm{~mA}(6), \mathrm{I}_{d}=90 \mathrm{~mA}(7)$, $\mathrm{I}_{d}=100 \mathrm{~mA}(8)$. Measurement error is equal to the diameter of the circle

The function $\mathrm{V}_{1}(\mathrm{H})$ is caused by the magnetic field dependence of the fractal dimension $\mathrm{D}(\mathrm{H})$, and thus the glassy state index $\mu$, the value of which in this case ranges from 1 to 2 . This corresponds to a creep bundles of vortices in the sample [15].

Two terms in formula (2) are necessary due to quantitative differences intragranular and intergranular differential resistance of the polycrystal. Distribution functions of defects on the areas and fractal dimension of their boundaries do not depend on whether they are inside of granules or between them.

The value $R_{d 1}=0.012 \mathrm{~m} \Omega$ in this approximation was reduced when compared to the value found in paper [8]. This is due to the lack of resistance for the values of the field strength of less than 50 Oe. Zero resistance in the beginning of the dependence $\mathrm{V}_{1}(\mathrm{H})$ is caused largely due to the formation of the hypervortices in the sample [17]. Therefore, the beginning of the increasing of voltage is determined by two processes: hypervortices decay into individual Josephson vortices and increase of fractality clusters of the normal phase with increasing field.

For the analysis of nonlinear dynamic systems can use the concept of nonequilibrium (quasi)stationary states with the topology of a percolating fractal set [18]. In this case, one of the main characteristics of the system is connectivity index $\theta$. At the percolation threshold connectivity index of the fractal can be identified by its fractal dimension:

$$
\theta=2\left(D / d_{s}-1\right)
$$

where $\mathrm{d}_{s}$ is the spectral fractal dimension.

Since at the percolation threshold $\mathrm{d}_{s}=\mathrm{C} \approx 1.327$ [18], it follows from (3) we can determine the values of the connectivity index for different values of the fractal dimension. In this case (Fig. 1) D varies from 1.2 to 1.9 , which corresponds to a change of the connectivity index $\theta$ from -0.2 to 1.43 . If $\mathrm{D}=\mathrm{C} \approx 1.327$, then $\theta$ changes sign from minus to plus, which means the transition from disconnected to connected systems, ie beginning of percolation. This value of the fractal dimension corresponds to the magnetic field strength $\approx 60-70$ Oe. This corresponds to the beginning vortex penetration into granules of polycrystalline YBCO. 


\section{References}

[1] M.Prester, Experimental evidence of a fractal dissipative regime in high- $\mathrm{T}_{c}$ superconductors, Phys. Rev. B, 60(1999), 3100-3103.

[2] D.A.Balaev, I.L.Belozerova, D.M.Gokhfeld, L.V.Kashkina, Yu.I.Kuzmin, C.R.Michel, M.I.Petrov, S.I.Popkov, K.A.Shaikhutdinov, Current-voltage characteristics of a foamed $\mathrm{Bi}_{1.8} \mathrm{~Pb}_{0.3} \mathrm{Sr}_{2} \mathrm{Ca}_{2} \mathrm{Cu}_{3} \mathrm{O}_{x}$ high-temperature superconductor with fractal cluster structure, Phys. Solid State, 48(2006), 207-212.

[3] R.Surdeanu, R.J.Wijngaarden, B.Dam, J.Rector, R.Griessen, C.Rossel, Z.F.Ren, J.H.Wang, Crossover between fractal and nonfractal flux penetration in high-temperature superconducting thin films, Phys. Rev. B, 58(1998), 12467-12477.

[4] M.Baziljevich, E.Baruch-El, T.H.Johansen, Y.Yeshurun, Dendritic instability in $\mathrm{YBa}_{2} \mathrm{Cu}_{3} \mathrm{O}_{7-x}$ films triggered by transient magnetic fields, Appl. Phys. Lett., 105(2014), 012602 .

[5] J.I.Vestgarden, P.Mikheenko, Y.M.Galperin, T.H.Johansen, Nonlocal electrodynamics of normal and superconducting films, New J. Phys., 15(2013,) 093001.

[6] A.V.Milovanov, J.J.Rasmussen, Fracton pairing mechanism for unconventional superconductors: Self-assembling organic polymers and copper-oxide compounds, Phys. Rev. B, 66(2002), 134505 .

[7] Yu.I.Kuzmin, Magnetic-flux creep at the initial stage of resistive transition in superconductors with fractal clusters of normal phase, Tech. Phys. Lett., 40(2014)], 769-772.

[8] M.A.Vasyutin, Fractal dimension of structural inhomogeneities in granular YBCO superconductor in magnetic field, Tech. Phys. Lett., 37(2011), 743-745.

[9] S.Kang, A.Goyal, J.Li, A.A.Gapud, P.M.Martin, L.Heatherly, J.R.Thompson, D.K.Christen, F.A.List, M.Paranthaman. D.F.Lee, High-performance high-Tc superconducting wires, Science, 311(2006), 1911-1914.

[10] Yu.I.Kuzmin, I.V.Pleshakov, S.V.Razumov, The statistical distribution of magnetic critical currents determined by HTSC film morfology, Phys. Solid State, 41(1999), 1594-1597.

[11] S.H.Wee, A.Goyal, E.D.Specht, C.Cantoni, Y.L.Zuev, V.Selvamanickam, Sy Cook, Enhanced flux pinning and critical current density via incorporation of self-assembled rare-earth barium tantalate nanocolumns within $\mathrm{YBa}_{2} \mathrm{Cu}_{3} \mathrm{O}_{7-\delta}$ films, Phys. Rev. B, 81,(2010)140503.

[12] Yu.I.Kuzmin, Resistive state of superconducting structures with fractal clusters of a normal phase, Phys. Solid State, 43(2001), 1199-1206.

[13] Yu.I.Kuzmin, Peculiarities of the resistive transition in fractal superconducting structures, Tech. Phys. Lett., 29(2003), 414-417.

[14] Yu.I.Kuzmin, Depinning at the initial stage of the resistive transition in superconductors with a fractal claster structure, Tech. Phys. Lett., 30(2004), 457-460.

[15] Yu.I.Kuzmin, Vortex glass state in superconductors with fractal clusters of normal phase, Tech. Phys. Lett., 36(2010), 400-403. 
[16] M.A.Vasyutin, N.D.Kuz'michev, Nonlinearity of the current-voltage characteristics of HTS $\mathrm{YBa}_{2} \mathrm{Cu}_{3} \mathrm{O}_{7-x}$, defined by a modulation technique. Tech. Phys. Lett., 18(1992), 764-766.

[17] N.D.Kuz'michev, Magnetic field penetration into the weak-link system of the $\mathrm{YBa}_{2} \mathrm{Cu}_{3} \mathrm{O}_{7-x}$ granular superconductor, Phys. Solid State, 43(2001), 2012-2017.

[18] L.M.Zelenyi, A.V.Milovanov, Fractal topology and strange kinetics from percolation theory to problems in cosmic electrodynamics, Phys. Usp., 47(2004), 749-788.

\section{Фрактальные границы кластеров пиннинга вихрей в меднооксидных сверхпроводниках в магнитном поле}

\section{Михаил А. Васютин \\ Николай Д. Кузьмичев \\ Дмитрий А.Шилкин}

Мордовский государственный университет им. Н. П. Огарёва Большевистская, 68, Саранск, 430005

Россия

Исследовано влияние фрактальных грании кластеров нормальной фазы на крип потока в меднооксидном высокотемпературном сверхпроводнике ҮВСО. Разработана модель магнитополевой зависимости напряжения, обусловленного крипом потока, для разных транспортных токов. Для описания экспериментальных зависимостей предложено использовать экспоненииальногиперболическую функиию, в которой параметром является ток. Определена эмпирическая зависимость фрактальной размерности грании, кластеров от напряжённости магнитного поля. Найдены характерные значения напряженности магнитного поля. Вычислен индекс связности путей срыва вихрей на пороге перколяиии.

Ключевые слова: сверхпроводимость, кластеры нормальной фазы, фрактальные границы, крип nотока. 